\title{
Exploring Bell nonlocality of quantum networks with stabilizing and logical operators
}

\author{
Li-Yi Hsu $\odot^{*}$ \\ Department of Physics, Chung Yuan Christian University, Chungli 32081, Taiwan \\ Ching-Hsu Chen (1) \\ Department of Electrophysics, National Chiayi University, Chiayi 600, Taiwan
}

(Received 8 October 2020; accepted 5 May 2021; published 24 May 2021)

\begin{abstract}
In practical quantum networks, a variety of multiqubit stabilized states emitted from independent sources are distributed among the agents, and the correlations across the entire network can be derived from each agent's local measurements on the shared composite quantum systems. To reveal the Bell nonlocality in such cases as a quantum feature, minimal knowledge of the emitted stabilizer state is required. Here, we demonstrate that knowing the stabilizing and logical operators indeed provides a way of exploring Bell nonlocality in quantum networks. For the qubit distribution in quantum networks, the associated nonlinear Bell inequalities are derived. On the other hand, to violate these inequalities, one can design local incompatible observables using minimal knowledge of the emitted states. The tilted nonlinear Bell inequalities tailored for specific nonmaximal entangled stabilizer states and a way of achieving the maximal violation are also explored.
\end{abstract}

DOI: 10.1103/PhysRevResearch.3.023139

\section{INTRODUCTION}

In the seminal work of Bell [1], it was shown that the quantum correlations arising from spatially separated systems can break the limits of classical causal relations [2]. In classical physics, the realism and locality of spacelike events constrain the strength of classical correlations bounded by the Bell inequalities. Quantum theory inconsistent with local realism predicts stronger correlations that violate the Bell inequalities. Thanks to quantum information science, two-particle and multiparticle quantum correlations have been extensively investigated. As a distinct feature from classical physics, Bell nonlocality has led to applications in quantum information processing including private random number generation [3], quantum cryptography [4], and reductions in communication complexity [5].

In typical Bell experiments on statistical correlations, a source emits a state comprising two or more particles that are shared between two or more distant observers, who each perform local measurements with a random chosen setting and then obtain the measurement outcomes. To reveal the Bell nonlocality of an entangled state, the local observables should be set deliberately. For example, to violate the Bell inequalities tailored to stabilizer states such as graph states, it is helpful to take the stabilizers as a reference for finding suitable measurement settings [6-8]. To emphasize the

\footnotetext{
*1yhsu@cycu.edu.tw

Published by the American Physical Society under the terms of the Creative Commons Attribution 4.0 International license. Further distribution of this work must maintain attribution to the author(s) and the published article's title, journal citation, and DOI.
}

role of stabilizing operators and logical bit-flip operators, we review the Clauser-Horne-Shimony-Holt (CHSH) inequality and its modification below. Let the two-qubit entangled state $\left(0<\phi<\frac{\pi}{2}\right)$

$$
|\phi\rangle=\cos \phi|\overline{0}\rangle+\sin \phi|\overline{1}\rangle,
$$

be the codeword of the $[2,1,2]$ stabilizer-based quantum error-detecting code with the logical states $|\overline{0}\rangle=|00\rangle$ and $|\overline{1}\rangle=|11\rangle$. The stabilizer generator, logical bit-flip, and phase-flip operators are $\sigma_{z} \otimes \sigma_{z}, \sigma_{x} \otimes \sigma_{x}$, and $I \otimes \sigma_{z}$, respectively $\left(\sigma_{x}, \sigma_{y}\right.$, and $\sigma_{z}$ are Pauli matrices, and $I$ is the identity operator). In the bipartite Bell test, the $\mathrm{CHSH}$ operator is $\mathbf{B}_{\mathrm{CHSH}}=\sum_{i, j=0}^{1}(-1)^{i j} A_{i} \otimes B_{j}$, where $A_{i}$ and $B_{j}$ are local observables, and the $\mathrm{CHSH}$ inequality states that $\left\langle\mathbf{B}_{\mathrm{CHSH}}\right\rangle \stackrel{c}{\leqslant} 2$, where the $\langle\cdot\rangle$ denotes the expectation value of $\cdot$. To violate the $\mathrm{CHSH}$ inequality, for the first-qubit, we assign

$$
\sigma_{z} \rightarrow \frac{1}{2 \cos \mu}\left(A_{0}+A_{1}\right), \quad \sigma_{x} \rightarrow \frac{1}{2 \sin \mu}\left(A_{0}-A_{1}\right),
$$

and for the second-qubit, we assign

$$
\sigma_{z} \rightarrow B_{0}, \quad \sigma_{x} \rightarrow B_{1} .
$$

It is easy to verify that

$$
\begin{aligned}
& \left\langle\phi\left|\mathbf{B}_{\mathrm{CHSH}}\right| \phi\right\rangle \\
& \quad=2 \cos \mu\left\langle\phi\left|\sigma_{z} \otimes \sigma_{z}\right| \phi\right\rangle+2 \sin \mu\left\langle\phi\left|\sigma_{x} \otimes \sigma_{x}\right| \phi\right\rangle \\
& \quad=2(\cos \mu+\sin \mu \sin 2 \phi) \\
& \quad=2 \sqrt{1+\sin ^{2} 2 \phi} \cos \left(\mu-\mu_{0}\right) \\
& \quad \leqslant 2 \sqrt{1+\sin ^{2} 2 \phi},
\end{aligned}
$$


where $\tan \mu_{0}=\sin 2 \phi$. Some remarks are in order. First, the operators $\left(A_{0}+A_{1}\right) \otimes B_{0}=\cos \mu \sigma_{z} \otimes \sigma_{z}$ and $\left(A_{0}-A_{1}\right) \otimes$ $B_{1}=\sin \mu \sigma_{x} \otimes \sigma_{x}$, and hence, both terms in the first equality in Eq. (4) exemplify the usefulness of the stabilizing operator and the logical (bit-flip) operator for finding the local observables that violate the $\mathrm{CHSH}$ inequality. The maximal $\mathrm{CHSH}$ value $>2$ can be achieved by setting $\mu=\mu_{0}$. Particularly, $\left|\phi=\frac{\pi}{4}\right\rangle$ is maximally entangled, $\sigma_{x} \otimes \sigma_{x}$ becomes another stabilizing operator rather than simply a logical bit-flip operator, and the CHSH inequality can be maximally violated. There are a variety of Bell inequalities violated by the graph states as a specific family of stabilizer states, where the associated Bell operators can be reformulated as the sum of their stabilizing operators, and hence, the perfect-antiperfect correlations therein can reach the maximal violation [7-12]. If the multiqubits mixed states involve two stabilizing operators, their nonlocality can be verified by the violation of stabilizerbased Bell-type inequalities [13,14]. Second, observables $A_{0}$ and $A_{1}$ can be regarded as the results of "cutting and mixing" the stabilizing operator and the logical bit-flip operator into the local observables:

$$
\begin{aligned}
\sigma_{z} & \otimes \sigma_{z}, \sigma_{x} \otimes \sigma_{x} \stackrel{\text { cutting }}{\longrightarrow} \sigma_{z}, \sigma_{x} \stackrel{\text { mixing }}{\longrightarrow} A_{x_{i}} \\
& =\cos \mu \sigma_{z}+(-1)^{x_{i}} \sin \mu \sigma_{x} .
\end{aligned}
$$

Cutting means cutting the first qubit observables from $\sigma_{z} \otimes$ $\sigma_{z}$ and $\sigma_{x} \otimes \sigma_{x}$; mixing means linearly superposing the two cut observables. In what follows, the local observables on the source side will be constructed in a similar way. In addition, note that the local observables anticommute; i.e., $\left\{A_{0}, A_{1}\right\}=\left\{B_{0}, B_{1}\right\}=0$. Third, the observable $B_{0}$ is the phase-flip operator, which can be exploited in the tilted $\mathrm{CHSH}$ operators $\mathbf{B}_{\beta \text {-CHSH }}=\beta B_{0}+\mathbf{B}_{\mathrm{CHSH}}$ with the Bell inequality $\left\langle\mathbf{B}_{\beta \text {-CHSH }}\right\rangle \stackrel{c}{\leqslant} 2+\beta$. By setting $\beta=2 / \sqrt{1+2 \tan ^{2} 2 \phi}$, the maximal violation can be achieved by $|\phi\rangle[15,16]$. That is, the nonmaximally entangled state can maximally violate the tilted Bell inequalities involving the phase-flip operators.

One can generalize the above approach to the multiqubit case. For example, let $|\psi\rangle=\cos \psi|\overline{0}\rangle+\sin \psi|\overline{1}\rangle$ be the codeword of the $[5,1,3]$ stabilizer-based quantum error-correcting code (SQECC), and let Alice hold the first qubit and Bob hold the other qubits. In this case, the useful stabilizing operator and logical bit-flip operator are $g=\sigma_{z} \otimes \sigma_{z} \otimes \sigma_{x} \otimes$ $I \otimes \sigma_{x}$ and $\sigma_{x}^{\otimes 5}$, respectively. The observables of the first and second qubits of the stabilizing operators and the bit-flip operator are the same as those in Eqs. (2) and (3), respectively. The last three qubits are termed idle qubits since the observable $\sigma_{x}$ is always measured on them, while the outcome of the fourth qubit is discarded if Bob measures $B_{1}^{\prime}$. In the Bell test, Alice randomly measures one of the observables in Eq. (2), whereas Bob randomly measures $B_{0}^{\prime}=$ $B_{0} \otimes \sigma_{x}^{\otimes 3}$ or $B_{1}^{\prime}=B_{1} \otimes \sigma_{x} \otimes I \otimes \sigma_{x}$. The CHSH-like inequality can be written as $\left\langle\sum_{i, j=0}^{1}(-1)^{i j} A_{i} \otimes B_{j}^{\prime}\right\rangle \stackrel{c}{c}$. As another example, let the logical states be $|\overline{0}\rangle=|0\rangle^{\otimes n}$ and $|\overline{1}\rangle=$ $|1\rangle^{\otimes n}$, and denote the $n$-qubit Greenberger-Horne-Zeilinger (GHZ) state $(n \geqslant 3)$ as $\left|\mathrm{GHZ}_{\phi}\right\rangle=\cos \phi|\overline{0}\rangle+\sin \phi|\overline{1}\rangle$. Alice holds the first $m$ qubits, and Bob holds the other $n-m$ qubits. Here, the useful stabilizing, logical bit-flip and phase-flip operators can be set as $\sigma_{z} \otimes I^{\otimes m-1} \otimes \sigma_{z} \otimes$
$I^{\otimes n-m-1}, \sigma_{x}^{\otimes n}$, and $I^{\otimes m} \otimes \sigma_{z} \otimes I^{\otimes n-m-1}$, respectively. One can construct the local observables $A_{i}^{\prime \prime}=\cos \mu \sigma_{z} \otimes I^{\otimes m-1}+$ $(-1)^{i} \sin \mu \sigma_{x}^{\otimes m}$ using the cutting-and-mixing scheme, and $B_{j}^{\prime \prime}=(1-j) \sigma_{z} \otimes I^{\otimes n-m-1}+j \sigma_{x}^{\otimes n-m}$ such that $\left\{A_{0}^{\prime \prime}, A_{1}^{\prime \prime}\right\}=$ $\left\{B_{0}^{\prime \prime}, B_{1}^{\prime \prime}\right\}=0$. Hence, we reach the CHSH-like inequality $\left\langle\sum_{i, j=0}^{1}(-1)^{i j} A_{i}^{\prime \prime} \otimes B_{j}^{\prime \prime}\right\rangle \stackrel{c}{\leqslant} 2$. Here, the last $n-m-1$ qubits are idle since only the observable $\sigma_{x}$ is always measured on each of them. Conditional on the qubit assignment, one can select the useful stabilizing operators and logical operators to derive similar CHSH-like inequalities.

Recently, Bell nonlocality in quantum networks like the generalized Bell experiments have attracted much research attention. Long-distance quantum networks of large-scale multi-users are the substantial goals of quantum communication, so it is fundamental to study their nonlocal capacities. A quantum network involves multiple independent quantum sources, where each of them initially emits a two- or multiqubit entangled state shared among a set of observers or agents. There are several obstacles to the study of nonlocality in a quantum network. The classical correlations of a network indicate more sophisticated causal relations and lead to stronger constraints than those in the typical (one-source) Bell scenario. In addition, each observer can perform a joint measurement on the qubits at hand, which could result in strong correlations across the network. Unlike the typical linear Bell-type inequalities, most Bell-type inequalities for various classes of networks are nonlinear, which implies the nonconvexity of the multipartite correlation space [17-20]. In the two-source case as the simplest quantum network, bilocal and nonlocal correlations were thoroughly investigated [21,22]. Next, the Bell-type inequalities for star-shaped and noncyclic networks were studied $[17,18]$. Recently, broader classes of quantum networks based on locally causal structures have also been investigated [19,20,23,24]. Computationally efficient algorithms for constructing Bell inequalities have been proposed [20]. These Bell-type inequalities are tailored for networks with quantum sources emitting either two-qubit Bell states or generalized GHZ states. The stabilizing operators and logical operators implicitly play substantial roles in setting up local joint observables. On the other hand, regarding the potential applications of encrypted communication in quantum networks, SQECCs can be more useful in quantum network cryptography, such as in quantum secret sharing [25] and secure quantum key distribution protocols [26]. Revealing Bell nonlocality in a network is required for detecting potential eavesdropping attacks. Moreover, a structured quantum state with stabilizing operators and logical operators is more useful in the engineering of quantum networks [27-31].

In this paper, we study the Bell nonlocality of quantum networks. Hereafter, we consider a $K$-locality network $\mathcal{N}$, as shown in Fig. 1. There are $K+M$ agents, of which $K$, $\mathcal{S}^{(1)} \ldots \mathcal{S}^{(K)}$ are on the source side and $M, \mathcal{R}^{(1)} \ldots \mathcal{R}^{(M)}$ on the receiver side. There are $N$ independent sources. Let $0=$ $e_{0}<e_{1}<\cdots<e_{K}=N$. The agent $\mathcal{S}^{(s)}$ holds the sources $e_{s-1}+1, \ldots$, and $e_{s}$; thus, the number of sources that $\mathcal{S}^{(s)}$ holds is $\left(e_{s}-e_{s-1}\right)$. The source $i\left(e_{s-1}+1 \leqslant i \leqslant e_{s}\right)$ held by $\mathcal{S}^{(s)}$ emits $n_{i}$ particles, of which $n_{i}^{(0)}(\neq 0)$ are in possession of the agent $\mathcal{S}^{(s)}$, and $n_{i}^{(m)}(\geqslant 0)$ are sent to the agent $\mathcal{R}^{(m)}$. Consequently, $\sum_{j=0}^{M} n_{i}^{(j)}=n_{i}$. In the classical networks, source $i$ 


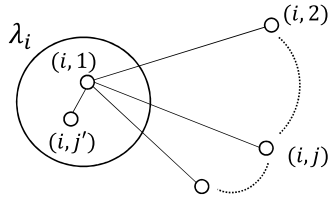

(a)

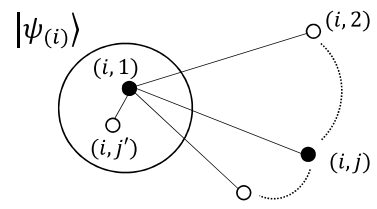

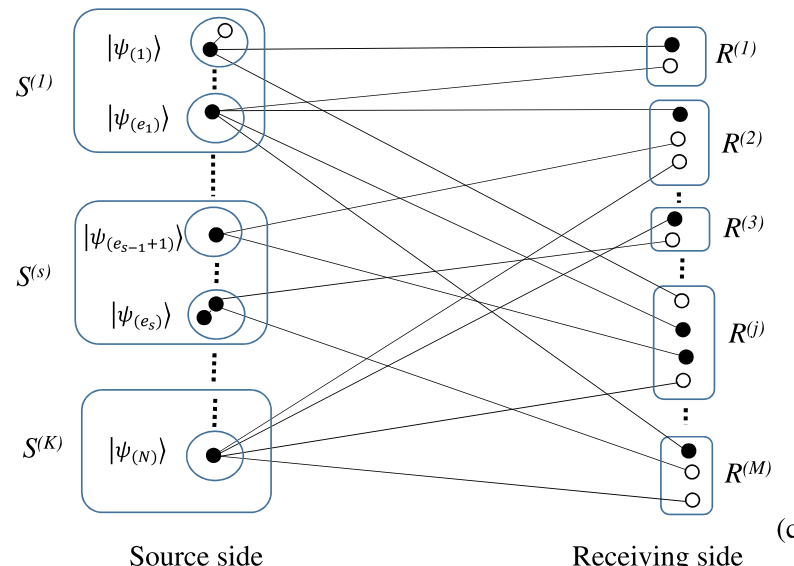

(c)

FIG. 1. The particle distribution of the state emitted from the source $i$ held by $\mathcal{S}^{(s)}$ if $e_{s-1}+1 \leqslant i \leqslant e_{s}$. The particles assigned on the source (receiving) side are inside (outside) the circle. The state can be (a) the classical hidden state $\lambda_{i}$ or (b) the quantum state $\left|\psi_{(i)}\right\rangle$. Here, the particle $(i, 1)$ assigned on the source side is determined as the center of the star graph. Qubit $(i, j)$ in (b) is denoted by a black dot if $\left\{\widehat{s}_{(i, j)}, \widehat{t}_{(i, j)}\right\}=0$. A quantum network is depicted in (c). The qubits in a rounded rectangle are locally accessible by an agent. According to Eqs. (28) and (29), there is an odd number of black dots in each rounded rectangle.

emits $n_{i}$ particles described by hidden state $\lambda_{i}$; in the quantum networks, it emits $n_{i}$ qubits in quantum state $\left|\psi_{(i)}\right\rangle$, which can be either a stabilizer state or a codeword of a SQECC. In the Bell test, observer $\mathcal{S}^{(s)}\left[\mathcal{R}^{(m)}\right]$ measures observable $A_{x_{s}}^{(s)}\left[B_{y_{m}}^{(m)}\right]$ with the associated outcome $a_{s}\left(b_{m}\right)$, where $x_{s}, y_{m} \in\{0,1\}$ and $a_{s}, b_{m} \in\{-1,1\}$. In the following, the index pair $(i, j)$ denotes the $j$ th particle emitted from source $i$, and $(i, j) \rightarrow \mathcal{X}^{(k)}$ indicates that particle $(i, j)$ is at agent $\mathcal{X}^{(k)}$ 's hand $(\mathcal{X} \in\{\mathcal{R}, \mathcal{S}\})$. Finally, we denote the particle sets $\mathbb{S}^{(k)}=\{(i, j) \mid \forall(i, j) \rightarrow$ $\left.\mathcal{S}^{(k)}\right\}$ and $\mathbb{R}^{(k)}=\left\{(i, j) \mid \forall(i, j) \rightarrow \mathcal{R}^{(k)}\right\}$.

The remainder of this paper is organized as follows: In Sec. II, we investigate the classical networks, where general local causal models (GLCMs) are introduced. Then Bell inequalities associated with classical networks are proposed. We study Bell nonlocality of $K$-locality quantum networks in Sec. III. First, we review the stabilizer states and SQECCs. Then we demonstrate how to violate the proposed Bell inequalities using deliberated local observables. It will be shown that the local observables can be made up of "cut-graftmixing" stabilizing operators and logical operators. Notably, there are two alternative nonlocal correlations. One is due to the entanglement of the logical states themselves, where two suitable stabilizing operators are exploited to construct the local observables. The other nonlocality results from the entanglement due to the superposition of logical states, in which a stabilizing operator and a logical operator can be suitably exploited in this case. We illustrate these two kinds of nonlocality in terms of the 5-qubit code, which is the smallest stabilizer code that protects against single-qubit errors. In Sec. IV, we propose Bell inequalities tailored for nonmaximally entangled states distributed in a quantum network. Finally, conclusions are drawn in Sec. V.

\section{CLASSICAL NETWORKS}

\section{A. GLCMs}

The GLCMs in classical networks can be described as follows: The $i$ th source is associated with a local random variable as the hidden state $\lambda_{i}$ in the measure space $\left(\Omega_{i}, \Sigma_{i}, \mu_{i}\right)$ with the normalization condition $\int_{\Omega_{i}} d \mu_{i}\left(\lambda_{i}\right)=1$. All systems in the Bell test scenario are considered in the hidden state $\Lambda=$ $\left(\lambda_{i}, \cdots, \lambda_{N}\right)$ in the measure space $(\Omega, \Sigma, \mu)$, where $\Omega=$ $\Omega_{1} \otimes \cdots \otimes \Omega_{N}$ and the measure of $\Lambda$ is given by $\mu(\Lambda)=$ $\prod \mu_{i}\left(\lambda_{i}\right)$ with the normalization condition $\int_{\Omega} d \mu(\Lambda)=1$. In the measurement phase, agent $\mathcal{S}^{(s)}$ performs the measurement $A_{x_{s}}^{(s)}$ on state $\Lambda_{S}^{(s)}$ with the corresponding outcome denoted by $a_{s} \in\{1,-1\}$. The GLCM suggests a joint conditional probability distribution of the measurement outcomes

$$
P(\mathbf{a} \mid \mathbf{x})=\int_{\Omega} d \mu(\Lambda) \prod_{s=1}^{K} P\left(a_{s} \mid x_{s}, \Lambda\right),
$$

where $\mathbf{a}=\left(a_{1}, a_{2}, \cdots, a_{K}\right)$ and $\mathbf{x}=\left(x_{1}, x_{2}, \cdots, x_{K}\right)$; hence, we have the correlation

$$
\left\langle A_{x_{1}}^{(1)} \ldots A_{x_{K}}^{(K)}\right\rangle=\sum_{\mathbf{a}} a_{1} \cdots a_{K} P(\mathbf{a} \mid \mathbf{x}, \Lambda) .
$$

On the other hand, in the $K$-locality condition [20], $\mathcal{S}^{(s)}$ can access the hidden state $\Lambda_{S}^{(s)}=\left(\lambda_{e_{s-1}+1}, \cdots, \lambda_{e_{s}}\right)$ in the measure space $\Omega_{S}^{(s)}=\Omega_{e_{s-1}+1} \otimes \cdots \otimes \Omega_{e_{s}}$, where

$$
\Lambda_{S}^{(s)} \cap \Lambda_{S}^{\left(s^{\prime}\right)}=\emptyset \Leftrightarrow s \neq s^{\prime},
$$

and

$$
\cup_{i=1}^{K} \Lambda_{S}^{(i)}=\Lambda
$$

Equation (6) can be rewritten as

$$
P(\mathbf{a} \mid \mathbf{x})=\prod_{s=1}^{K} \int_{\Omega_{S}^{(s)}} d \mu_{S}\left[\Lambda_{S}^{(s)}\right] P\left[a_{s} \mid x_{s}, \Lambda_{S}^{(s)}\right] .
$$

Denote the local expectation as

$$
\left\langle A_{x_{s}}^{(s)}\right\rangle=\sum_{a_{s}=-1,1} P\left[a_{S} \mid x_{s}, \Lambda_{S}^{(s)}\right] .
$$

By $K$ locality with the GLCM, we have

$$
\left\langle A_{x_{1}}^{(1)} \ldots A_{x_{K}}^{(K)}\right\rangle=\prod_{s=1}^{K}\left\langle A_{x_{s}}^{(s)}\right\rangle .
$$


Denote $\Delta^{ \pm} A^{(i)}=\frac{1}{2}\left[\left\langle A_{0}^{(i)}\right\rangle \pm\left\langle A_{1}^{(i)}\right\rangle\right]$. Since $-1 \leqslant\left\langle A_{x_{i}}^{(i)}\right\rangle \leqslant 1$, we have

$$
-1 \leqslant \Delta^{-} A^{(i)}, \quad \Delta^{+} A^{(i)} \leqslant 1,
$$

and

$$
\left|\Delta^{+} A^{(i)}\right|+\left|\Delta^{-} A^{(i)}\right|=\max \left\{\left|\left\langle A_{0}^{(i)}\right\rangle\right|,\left|\left\langle A_{1}^{(i)}\right\rangle\right|\right\} \leqslant 1 .
$$

On the receiving side, let $n_{j}^{(m)}>0$ if $j \in\left\{j_{1}, \cdots, j_{k_{m}}\right\}$ and $n_{j}^{(m)}=0$ otherwise. In this case, $\mathcal{R}^{(j)}$ receives the hidden states $\Lambda_{R}^{(m)}=\left(\lambda_{j_{1}}, \cdots, \lambda_{j_{k_{m}}}\right)$ in the measure space $\Omega_{R}^{(m)}=\Omega_{j_{1}} \otimes \cdots \otimes \Omega_{j_{k_{m}}}$, where $1 \leqslant j_{1}<j_{2} \ldots<j_{k_{m}} \leqslant N$. In the measurement phase, $\mathcal{R}^{(m)}$ performs the measurement $B_{y_{m}}^{(m)}, y_{m} \in\{0,1\}$, on the state $\Lambda_{R}^{(m)}$ with the corresponding outcome denoted by $b_{m} \in\{1,-1\}$. We have

$$
\left|\left\langle B_{y_{m}}^{(m)}\right\rangle\right|=\left|\int_{\Omega_{R}^{(m)}} \prod_{k} d \mu_{k}\left(\lambda_{k}\right) \sum_{b_{m}=-1,1} b_{m} P\left[b_{m} \mid y_{m}, \Lambda_{R}^{(m)}\right]\right| \leqslant 1 .
$$

\section{B. Bell inequalities}

The correlation strength in the proposed $K$-locality network is evaluated by two quantities:

$$
\mathbf{I}_{K, M}=\frac{1}{2^{K}}\left\langle\prod_{i=1}^{K} \prod_{j=1}^{M}\left[A_{0}^{(i)}+A_{1}^{(i)}\right] B_{0}^{(j)}\right\rangle,
$$

and

$$
\mathbf{J}_{K, M}=\frac{1}{2^{K}}\left\langle\prod_{i=1}^{K} \prod_{j=1}^{M}\left[A_{0}^{(i)}-A_{1}^{(i)}\right] B_{1}^{(j)}\right\rangle .
$$

In the classical scenario, we have

$$
\begin{aligned}
& \left|\mathbf{I}_{K, M}\right|_{\mathrm{GLCM}} \\
& =\frac{1}{2^{K}} \int_{\Omega} \prod_{i=1}^{K}\left|\left\langle A_{0}^{(i)}+A_{1}^{(i)}\right\rangle\right| \prod_{j=1}^{M}\left|\left\langle B_{0}^{(j)}\right\rangle\right| \prod_{k=1}^{N} d \mu_{k}\left(\lambda_{k}\right) \\
& \quad \leqslant \int_{\Omega} \prod_{i=1}^{K}\left|\Delta^{+} A^{(i)}\right| \prod_{k=1}^{N} d \mu_{k}\left(\lambda_{k}\right),
\end{aligned}
$$

and

$$
\begin{aligned}
& \left|\mathbf{J}_{K, M}\right|_{\mathrm{GLCM}} \\
& =\frac{1}{2^{K}} \int_{\Omega} \prod_{i=1}^{K}\left|\left\langle A_{0}^{(i)}-A_{1}^{(i)}\right\rangle\right| \prod_{j=1}^{M}\left|\left\langle B_{1}^{(j)}\right\rangle\right| \prod_{k=1}^{N} d \mu_{k}\left(\lambda_{k}\right) \\
& \quad \leqslant \int_{\Omega} \prod_{i=1}^{K}\left|\Delta^{-} A^{(i)}\right| \prod_{k=1}^{N} d \mu_{k}\left(\lambda_{k}\right),
\end{aligned}
$$

where the inequalities are from Eq. (15). Before proceeding further, two useful lemmas are introduced as follows:

Lemma 1 (Mahler inequality). Let $\alpha_{k}$ and $\beta_{k}$ be nonnegative real numbers, and let $p \in \mathbb{N}$; then

$$
\prod_{k=1}^{p} \alpha_{k}^{1 / p}+\prod_{k=1}^{p} \beta_{k}^{1 / p} \leqslant \prod_{k=1}^{p}\left(\alpha_{k}+\beta_{k}\right)^{1 / p} .
$$

The proof can be found in Ref. [17].

We obtain the following nonlinear Bell inequality:

$$
\begin{aligned}
& \left|\mathbf{I}_{K, M}\right|_{\mathrm{GLCM}}^{\frac{1}{K}}+\left|\mathbf{J}_{K, M}\right|_{\mathrm{GLCM}}^{\frac{1}{K}} \\
& \quad \leqslant\left\{\int_{\Omega} \prod_{i=1}^{K}\left[\left|\Delta^{+} A^{(i)}\right|+\left|\Delta^{-} A^{(i)}\right|\right] \prod_{k=1}^{N} d \mu_{k}\left(\lambda_{k}\right)\right\}^{\frac{1}{K}} \\
& \quad=\left\{\int_{\Omega} \prod_{i=1}^{K}\left(\max \left\{\left|\left\langle A_{x_{i}=0}\right\rangle\right|,\left|\left\langle A_{x_{i}=1}\right\rangle\right|\right\}\right) \prod_{k=1}^{N} d \mu_{k}\left(\lambda_{k}\right)\right\}^{\frac{1}{K}} \\
& \quad \leqslant\left[\int_{\Omega} \prod_{j=k}^{N} d \mu_{k}\left(\lambda_{k}\right)\right]^{\frac{1}{K}}=1,
\end{aligned}
$$

where the first inequality is from Lemma 1, and the fourth line is a consequence of Eq. (14).

\section{BELL NONLOCALITY OF A QUANTUM NETWORK}

\section{A. Review of stabilizer states and SQECC}

Let the state $\left|\psi_{(i)}\right\rangle$ emitted from the quantum source $i$ be an $n_{i}$-qubit stabilizer state. By definition, an $n_{i}$-qubit stabilizer state is one that is stabilized by a stabilizer which is a nontrivial subgroup of the Pauli group. If $\left|\psi_{(i)}\right\rangle$ as a codeword of $\left[n_{i}, k_{i}, d_{i}\right]$ SQECC, denote the last $\left(k_{i}\right.$ th) logical qubit as $\left|\overline{0}_{i}\right\rangle$ and $\left|\overline{1}_{i}\right\rangle$ and the corresponding logical bit- and phase-flip operators as $\bar{X}_{(i)}$ and $\bar{Z}_{(i)}$. Without loss of generality, we have

$$
\left|\psi_{(i)}\right\rangle=\sum_{z \in\{0,1\}^{\otimes k_{i}}} a_{z}|\bar{z}\rangle=\cos \phi_{i}\left|\varphi_{i}^{0}\right\rangle\left|\overline{0}_{i}\right\rangle+\sin \phi_{i}\left|\varphi_{i}^{1}\right\rangle\left|\overline{1}_{i}\right\rangle,
$$

where $\left\langle\varphi_{i}^{0} \mid \varphi_{i}^{1}\right\rangle \in R$, and $\left\langle\varphi_{i}^{0} \mid \varphi_{i}^{0}\right\rangle=\left\langle\varphi_{i}^{1} \mid \varphi_{i}^{1}\right\rangle=1$. In addition,

$$
\begin{gathered}
\bar{X}_{(i)}\left|\overline{0}_{i}\right\rangle=\left|\overline{1}_{i}\right\rangle, \quad \bar{X}_{(i)}\left|\overline{1}_{i}\right\rangle=\left|\overline{0}_{i}\right\rangle, \\
\bar{Z}_{(i)}\left|\bar{c}_{i}\right\rangle=(-1)^{c}\left|\bar{c}_{i}\right\rangle(c \in\{0,1\}) .
\end{gathered}
$$

In what follows, we exploit $g_{(i)}$ and $g_{(i)}^{\prime}$ as useful stabilizing operators, and $\bar{X}_{(i)}$ and $\bar{Z}_{(i)}$ as useful logical operators. Denote the Pauli set of qubit $(i, j)$ as $\mathbb{P}_{(i, j)}=\left\{X_{(i, j)}, Y_{(i, j)}, Z_{(i, j)}\right.$, $\left.I_{(i, j)}\right\}$, where $X_{(i, j)}=\sigma_{x}, Y_{(i, j)}=\sigma_{y}, Z_{(i, j)}=\sigma_{z}$, and $I_{(i, j)}=$ $I$. Let $h_{(i)} \in\left\{\bar{X}_{(i)}, g_{(i)}^{\prime}\right\}$ and $h_{(i)}^{\prime} \in\left\{\bar{X}_{(i)}, \bar{Z}_{(i)}\right\}$. Note that

$$
\left[g_{(i)}, h_{(i)}\right]=\left[g_{(i)}, h_{(i)}^{\prime}\right]=0,
$$

and since $g_{(i)}, h_{(i)}$, and $h_{(i)}^{\prime}$ are $n_{i}$ fold tensor products of the Pauli operators, we have

$$
g_{(i)}=\prod_{j=1}^{n_{i}} \widehat{s}_{(i, j)}, h_{(i)}=\prod_{j=1}^{n_{i}} \widehat{t}_{(i, j)}, h_{(i)}^{\prime}=\prod_{j=1}^{n_{i}} \widehat{t}_{(i, j)}^{\prime},
$$

where $\widehat{s}_{(i, j)}, \widehat{t}_{(i, j)}$, and $\widehat{t}_{(i, j)} \in \mathbb{P}_{(i, j)} \forall i, j$.

Before proceeding further, some notations are introduced as follows: Denote the qubit index sets as $\mathbb{D}_{i}=\left\{j \mid\left\{\widehat{s}_{(i, j)}, \widehat{t}_{(i, j)}\right\}=0\right\}$ and $\mathbb{H}_{i}=\left\{j \mid\left[\widehat{s}_{(i, j)}, \widehat{t}_{(i, j)}\right]=0\right\}$, where $\mathbb{D}_{i} \cap \mathbb{H}_{i}=\emptyset$, and $\left|\mathbb{D}_{i}\right|+\left|\mathbb{H}_{i}\right|=n_{i}$. The qubits belonging to the sets $\mathbb{D}_{1}, \cdots, \mathbb{D}_{N}$ play substantial roles in the proposed Bell inequalities of the quantum networks. Note that qubit $(i, j)$ is called idle if $(i, j) \in \mathbb{H}_{i}$ and $(i, j) \rightarrow \mathcal{R}^{(k)}$ for some $k$, and the nonidentity operator 
of $\widehat{s}_{(i, j)}$ or $\widehat{t}_{(i, j)}$ is denoted by $\widehat{o}_{(i, j)}$, which will be repeatedly measured on qubit $(i, j)$ in the Bell test. Let the indicator $\delta_{(i, j)}^{D}=1$ if qubit $(i, j) \in \mathbb{D}_{i}$, and 0 if $(i, j) \in \mathbb{H}_{i}$. According to Eq. (25), we have $\sum_{j=1}^{n_{i}} \delta_{(i, j)}^{D} \bmod 2=0$, and hence,

$$
\sum_{i=1}^{N} \sum_{j=1}^{n_{i}} \delta_{(i, j)}^{D} \bmod 2=0
$$

Here, we focus on quantum networks fulfilling the following conditions: Regarding the qubits held by $\mathcal{S}^{(k)}$,

$$
\sum_{i=e_{k-1}+1}^{e_{k}} \sum_{j=1,(i, j) \in \mathbb{S}^{(k)}}^{n_{i}} \delta_{(i, j)}^{D} \bmod 2=1 \forall k=1,2, \cdots, K,
$$

and regarding the qubits held by $\mathcal{R}^{(k)}$,

$$
\sum_{i, j,(i, j) \in \mathbb{R}^{(k)}} \delta_{(i, j)}^{D} \bmod 2=1 \forall j=1, \cdots, M .
$$

Combining the constraints in Eqs. (27)-(29), the value $K+M$ must be even. According to the qubit distribution, it is very flexible to choose suitable $g_{(i)}, h_{(i)}$, and $h_{(i)}^{\prime}$ to implement local observables.

\section{B. Violation of the Bell inequalities in a quantum network}

To implement the local observables on the source side, we assign

$$
\widehat{S}^{(k)}=\prod_{i, j,(i, j) \in \mathbb{S}^{(k)}} \widehat{s}_{(i, j)} \rightarrow \frac{1}{2 \cos \mu}\left[A_{0}^{(k)}+A_{1}^{(k)}\right],
$$

and

$$
\widehat{T}^{(k)}=\prod_{i^{\prime}, j^{\prime},\left(i^{\prime}, j^{\prime}\right) \in \mathbb{S}^{(k)}} \widehat{t}_{\left(i^{\prime}, j^{\prime}\right)} \rightarrow \frac{1}{2 \sin \mu}\left[A_{0}^{(k)}-A_{1}^{(k)}\right] .
$$

Since $A_{x_{k}}^{(k)}=A_{x_{k}}^{(k) \dagger}$ and $\left[A_{x i}^{(i)}\right]^{2}=A_{x_{i}}^{(i)} A_{x i}^{(i) \dagger}=I, A_{x_{k}}^{(k)}$ is a unitary Hermitian with an eigenvalue of either 1 or -1 . On the receiving side, the local observables $B_{y_{l}}^{(l)}$ for observer $\mathcal{R}^{(k)}$ are

$$
B_{y_{l}}^{(l)}=\left(1-y_{l}\right) \prod_{i, j,(i, j) \in \mathbb{R}^{(l)}} \widehat{\boldsymbol{s}}_{(i, j)}+y_{l} \prod_{i^{\prime}, j^{\prime},\left(i^{\prime}, j^{\prime}\right) \in \mathbb{R}^{(l)}} \widehat{t}_{\left(i^{\prime}, j^{\prime}\right)},
$$

where $\left\{B_{0}^{(k)}, B_{1}^{(k)}\right\}=0$ according to Eq. (29). In the measuring phase, $\mathcal{S}^{(k)}\left[\mathcal{R}^{(k)}\right]$ randomly measures either the observable $A_{0}^{(k)}$ or $A_{1}^{(k)}\left[B_{0}^{(k)}\right.$ or $\left.B_{1}^{(k)}\right]$ with an outcome of either 1 or -1 . In practice, if the qubit $\left(i^{\prime \prime}, j^{\prime \prime}\right) \rightarrow \mathcal{R}^{(k)}$ is idle, $\mathcal{R}^{(k)}$ can always measure the observable $\widehat{o}_{\left(i^{\prime \prime}, j^{\prime \prime}\right)}$ in each round and discard the outcome in postprocessing if $\widehat{s}_{\left(i^{\prime \prime}, j^{\prime \prime}\right)}=I$ or $\widehat{t}_{\left(i^{\prime \prime}, j^{\prime \prime}\right)}=I$.

To evaluate the correlation strength, let $|\bar{\Psi}\rangle=$ $\prod_{i}^{N} \otimes\left|\psi_{(i)}\right\rangle$, and we have

$$
\begin{aligned}
\left|\mathbf{I}_{K, M}\right|_{Q} & =\frac{1}{2^{K}}\left|\sum_{x_{1}, \cdots x_{M}=0}^{1}\left\langle A_{x_{1}}^{(1)} \cdots A_{x_{K}}^{(K)} \prod_{j=1}^{M} B_{0}^{(j)}\right\rangle\right| \\
& =\frac{1}{2^{K}}\left|\left\langle\prod_{i=1}^{K}\left[A_{0}^{(i)}+A_{1}^{(i)}\right] \prod_{j=1}^{M} B_{0}^{(j)}\right\rangle\right|
\end{aligned}
$$

$$
\begin{gathered}
=\mid\left\langle\prod_{k=1}^{K} \cos \theta_{k} \prod_{i, j,(i, j) \in \mathbb{S}^{(k)}} \widehat{s}_{(i, j)}\right. \\
\left.\times \prod_{l=1}^{M} \prod_{i^{\prime}, j^{\prime},\left(i^{\prime}, j^{\prime}\right) \in \mathbb{R}^{(l)}} \widehat{s}_{\left(i^{\prime}, j^{\prime}\right)}\right\rangle \mid \\
=\left|\prod_{k=1}^{K} \cos \theta_{k}\left\langle\prod_{i=1}^{N} g_{(i)}\right\rangle\right| \\
=\left|\prod_{k=1}^{K} \cos \theta_{k}\right|,
\end{gathered}
$$

where $\langle\cdot\rangle=\langle\bar{\Psi}|\cdot| \bar{\Psi}\rangle$, and hence, $\left\langle\prod_{i=1}^{N} g_{(i)}\right\rangle=1 . \quad$ In addition,

$$
\begin{aligned}
\left|\mathbf{J}_{K, M}\right|_{Q}= & \frac{1}{2^{K}}\left|\sum_{x_{1}, \cdots x_{M}=0}^{1}(-1)^{\sum x_{j}}\left\langle A_{x_{1}}^{(1)} \cdots A_{x_{n}}^{(n)} \prod_{j=1}^{M} B_{1}^{(j)}\right\rangle\right| \\
= & \frac{1}{2^{K}}\left|\left\langle\prod_{i=1}^{K}\left[A_{0}^{(i)}-A_{1}^{(i)}\right] \prod_{j=1}^{M} B_{1}^{(j)}\right\rangle\right| \\
= & \mid\left\langle\prod_{k=1}^{K} \sin \theta_{k} \prod_{i, j,(i, j) \in \mathbb{S}^{(k)}} \widehat{t}_{(i, j)}\right. \\
& \left.\times \prod_{l=1}^{M} \prod_{i^{\prime}, j^{\prime},\left(i^{\prime}, j^{\prime}\right) \in \mathbb{R}^{(l)}} \widehat{t}_{\left(i^{\prime}, j^{\prime}\right)}\right\rangle \mid \\
& =\left|\prod_{j=1}^{K} \sin \theta_{j} \prod_{i=1}^{N}\left\langle h_{(i)}\right\rangle\right| .
\end{aligned}
$$

A useful lemma is introduced as follows:

Lemma 2 . For any $\theta_{1}, \theta_{2}, \cdots, \theta_{K} \in\left[0, \frac{\pi}{2}\right]$,

$$
\left|\prod_{j=1}^{K} \sin \theta_{j}\right|^{\frac{1}{K}} \leqslant \sin \bar{\theta} \text { and }\left|\prod_{j=1}^{K} \cos \theta_{j}\right|^{\frac{1}{K}} \leqslant \cos \bar{\theta}
$$

where $\bar{\theta}=\frac{1}{K} \sum_{j=1}^{K} \theta_{j}$. The proof can be found in Ref. [20].

Let $c_{i}=\left\langle h_{(i)}\right\rangle \leqslant 1$ and $C=\left|\prod_{i=1}^{N} c_{i}\right|^{\frac{1}{K}}$. We have

$$
\begin{aligned}
& \left|\mathbf{I}_{K, M}\right|_{Q}^{\frac{1}{K}}+\left|\mathbf{J}_{K, M}\right|_{Q}^{\frac{1}{K}} \\
& \quad=\left|\prod_{k=1}^{K} \cos \theta_{j}\right|^{\frac{1}{K}}+\left|\prod_{k=1}^{K} \sin \theta_{j}\right|^{\frac{1}{K}}|C| \\
& \quad \leqslant|\cos \bar{\theta}|+|\sin \bar{\theta}||C| \\
& \quad \leqslant \sqrt{1+C^{2}},
\end{aligned}
$$

where the first inequality in Eq. (36) follows from Lemma 2 , and the second inequality follows from the CauchySchwarz inequality. To reach the maximal violation, by setting 
$\theta_{1}=\ldots=\theta_{K}=\bar{\theta}$, and $\tan \bar{\theta}=C$, we obtain

$$
\max \left(\left|\mathbf{I}_{K, M}\right|_{Q}^{\frac{1}{K}}+\left|\mathbf{J}_{K, M}\right|_{Q}^{\frac{1}{K}}\right)=\sqrt{1+C^{2}}>1 .
$$

Some discussion is in order. The state $\left|\psi_{(i)}\right\rangle$ can contribute to the nonlocality of the quantum network through different facets of its nonlocality. Loosely speaking, there are $C_{2}^{2^{n_{i}-k_{i}}}$ ways of choosing suitable $g_{(i)}$ and $g_{(i)}^{\prime}$ and $k_{i}\left[2^{2\left(n_{i}-k_{i}\right)}\right]$ ways of choosing suitable $g_{(i)}$ and $\bar{X}_{(i)}$, which reflect the flexibility of testing Bell nonlocality in the quantum networks. The maximum value of $C=1\left(\bar{\theta}=\frac{\pi}{4}\right)$, and hence, $\max \left(\left|I_{K, M}\right|_{Q}^{\frac{1}{K}}+\left|J_{K, M}\right|_{Q}^{\frac{1}{K}}\right)=\sqrt{2}$, can be reached if $c_{i}=1 \forall i$. That is, $h_{(i)}$ stabilizes $\left|\psi_{(i)}\right\rangle \forall i$. As shown in Eqs. (30) and (31), one can benefit from the stabilizing operators in designing local observables to achieve the maximal violations of Bell inequalities in $K$-locality quantum networks. In detail, $c_{i}=1$ if $h_{(i)}=\bar{X}_{(i)},\left|\left\langle\varphi_{i}^{0} \mid \varphi_{i}^{1}\right\rangle\right|=1$, and $\phi_{i}=\frac{\pi}{4}$. The Bell nonlocality here is due to the superposition of $\left|\overline{0}_{i}\right\rangle$ and $\left|\overline{1}_{i}\right\rangle$; if $h_{(i)}=g_{(i)}^{\prime}, c_{i}$ is certain to be 1 . The Bell nonlocality in a network is due to that of either the stabilizer state or the logical states of the stabilizer code $\left[n_{i}, k_{i}, d_{i}\right]$. Note that such nonlocality involving the stabilizing operators $g_{(i)}$ and $g_{(i)}^{\prime}$ can be also obtained even by using specific states stabilized by the same stabilizing operators. For example, given source $i$ emitting a 4-qubit state with stabilizing operators $g_{(i)}=\sigma_{z}^{\otimes 4}$ and $g_{(i)}^{\prime}=\sigma_{x}^{\otimes 4}$, which both can also stabilize the 4-qubit Smolin state $\rho_{\text {Smolin }}=\frac{1}{4} \sum_{i j=0,1}\left|\Psi_{i j}\right\rangle\left\langle\Psi_{i j}\right|$, where $\left|\Psi_{i j}\right\rangle=\sigma_{z}^{i} \otimes \sigma_{x}^{j}\left[\frac{1}{\sqrt{2}}(|00\rangle+|11\rangle)\right]$. Eventually, Bell nonlocality of at most $\left|\mathbb{D}_{i}\right|$-qubit entanglement in $\left|\psi_{(i)}\right\rangle$ is involved.

As an illustration, let $K=N=2$ and $M=1$, and let sources 1 and 2 each emit the codeword states of $[5,1,3]$ SQECC with four stabilizer generators [the subscript qubit index $(i, j)$ is shortened as $j]$

$$
\begin{array}{ll}
\mathfrak{g}_{1}=X_{1} Z_{2} Z_{3} X_{4} I_{5}, & \mathfrak{g}_{2}=I_{1} X_{2} Z_{3} Z_{4} X_{5}, \\
\mathfrak{g}_{3}=X_{1} I_{2} X_{3} Z_{4} Z_{5}, & \mathfrak{g}_{4}=Z_{1} X_{2} I_{3} X_{4} Z_{5} .
\end{array}
$$

The well-known logical bit-flip and phase-flip operators are $\bar{X}^{\prime}=\prod_{i=1}^{5} X_{i}$ and $\bar{Z}^{\prime}=\prod_{i=1}^{5} Z_{i}$. Observers $\mathcal{S}^{(1)}$ and $\mathcal{S}^{(2)}$ hold qubits $(1,1)$ and $(2,1)$, respectively, while observer $\mathcal{R}^{(k)}$ holds the other 8 qubits, as shown in Fig. 2. Note that, in the following examples, the bilocal inequality is

$$
\sqrt{\mathbf{I}_{2,1}}+\sqrt{\mathbf{J}_{2,1}} \leqslant 1 \text {, }
$$

which is exactly the bilocal inequality for binary inputs and outputs in Ref. [21].

Example (a). Let $\left|\psi_{(1)}\right\rangle=\left|\psi_{(2)}\right\rangle=\cos \phi|\overline{0}\rangle+\sin \phi|\overline{1}\rangle$. Here, we choose the useful operators $g_{(i)}=\mathfrak{g}_{1} \mathfrak{g}_{2} \mathfrak{g}_{3} \mathfrak{g}_{4}=$ $Z_{1} Z_{2} X_{3} I_{4} X_{5}$ and $h_{(i)}=\bar{X}=\bar{X}^{\prime}$. In this case, $\left|\mathbb{D}_{i}\right|=2$, and $(i, 3),(i, 4),(i, 5)$ are idle qubits. Here, $A_{x_{i}}^{(i)}=$ $\frac{1}{\sqrt{2}}\left[Z_{(i, 1)}+(-1)^{x_{i}} X_{(i, 1)}\right], \quad B_{0}^{(1)}=\prod_{i=1}^{2} Z_{(i, 2)} X_{(i, 3)} X_{(i, 5)}, \quad$ and $B_{1}^{(1)}=\prod_{i=1}^{2} X_{(i, 2)} X_{(i, 3)} X_{(i, 4)} X_{(i, 5)}$. Note that $\mathbf{I}_{2,1}$ and $\mathbf{J}_{2,1}$ here are formally equivalent to $I^{22}$ and $J^{22}$ in Ref. [21]. Denote the 5-qubit state $|\varphi\rangle=\left(\cos \phi|0\rangle_{1}|0\rangle_{2}+\right.$ $\left.\sin \phi|1\rangle_{1}|1\rangle_{2}\right)|+\rangle_{3}|+\rangle_{4}|+\rangle_{5}$. It is easy to verify that

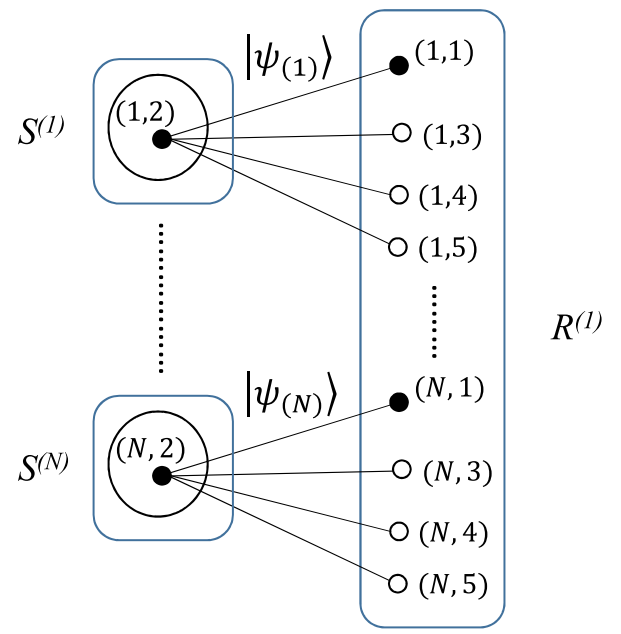

FIG. 2. A starlike quantum network with all states $\left|\psi_{(1)}\right\rangle, \ldots,\left|\psi_{(N)}\right\rangle$ being $[5,1,3]$ codeword states.

$g_{(i)}|\varphi\rangle=|\varphi\rangle$ and $\left\langle\psi_{(i)}\left|h_{(i)}\right| \psi_{(i)}\right\rangle=\left\langle\varphi\left|h_{(i)}\right| \varphi\right\rangle$. As a result, the same violation can be obtained using either $\left|\psi_{(1)}\right\rangle\left|\psi_{(2)}\right\rangle$ or $|\varphi\rangle^{\otimes 2}$.

Example (b). Let $\left|\psi_{(1)}\right\rangle=|\overline{0}\rangle,\left|\psi_{(2)}\right\rangle=|\overline{1}\rangle$. Here, we choose the useful operators $g_{(i)}=\mathfrak{g}_{1} \mathfrak{g}_{2} \mathfrak{g}_{3} \mathfrak{g}_{4}=Z_{1} Z_{2} X_{3} I_{4} X_{5}$ and $h_{(i)}=\mathfrak{g}_{1}$ for any $i \in\{1,2\}$. In this case, the local observables $A_{x_{i}}^{(i)}$ and $B_{0}^{(1)}$ are the same as those in example (a), while $B_{1}^{(1)}=\prod_{i=1}^{2} Z_{(i, 2)} Z_{(i, 3)} X_{(i, 4)}$. Here, $\left|\mathbb{D}_{i}\right|=2$ and $(i, 2),(i, 4)$, $(i, 5)$ are idle qubits. However, note that $g_{(i)}\left|\varphi^{\prime}\right\rangle=h_{(i)}\left|\varphi^{\prime}\right\rangle=$ $\left|\varphi^{\prime}\right\rangle$, where $\left|\varphi^{\prime}\right\rangle=\frac{1}{\sqrt{2}}\left(|0\rangle_{1}|+\rangle_{3}+|1\rangle_{1}|-\rangle_{3}\right)|0\rangle_{2}|+\rangle_{4}|+\rangle_{5}$. The maximal violation can be obtained using either $\left|\psi_{(1)}\right\rangle\left|\psi_{(2)}\right\rangle$ or $\left|\varphi^{\prime}\right\rangle^{\otimes 2}$.

Consequently, although the two 5-qubit logical states $|\overline{0}\rangle$ and $|\overline{1}\rangle$ and the codeword states are genuinely entangled $[32,33]$, one can replace the genuinely entangled state with a state involving 2-qubit entanglement, either $|\varphi\rangle$ or $\left|\varphi^{\prime}\right\rangle$, to reach the same correlation strength. It is not known whether the Bell nonlocality of genuine entanglement can be revealed in a $K$-locality quantum network. Specifically, it is not known whether the genuine entanglement of $\left|\psi_{(i)}\right\rangle$ can be deduced from violations of variant Bell inequalities of a quantum network involving different stabilizing operators.

\section{BELL INEQUALITIES TAILORED FOR NONMAXIMAL ENTANGLED STATES IN A QUANTUM NETWORK}

If $c_{i}<1$ for at least one $i,|\bar{\Psi}\rangle$ cannot achieve the maximal violation of the nonlinear Bell inequality in Eq. (21). To explore the Bell inequalities maximally violated by $|\bar{\Psi}\rangle$, recall that, in the 2-qubit case $\left(N=K=M=1, n_{1}=2\right)$, the tilted $\mathrm{CHSH}$ operator $\mathbf{B}_{\beta-\mathrm{CHSH}}=\beta B_{0}+\mathbf{B}_{\mathrm{CHSH}}$ is exploited using the logical phase-flip operator $B_{0}$ with appropriate parameter $\beta$. Although it is unlikely that $\prod_{m=1}^{M} B_{0}^{(m)}=\prod_{i=1}^{N} \bar{Z}_{(i)}$ in quantum networks, it will be shown that the logical phase-flip operators are still useful in finding the tilted Bell inequalities. Denote two index sets $\mathfrak{C}=\left\{i \mid c_{i}=1\right\}$ and $\mathfrak{c}=\left\{i^{\prime} \mid c_{i^{\prime}}<1\right\}$, where $\mathfrak{C} \cap \mathfrak{c}=\emptyset$ and $|\mathfrak{C}|+|\mathfrak{c}|=N$. Without loss of generality, let $i \in \mathfrak{C}$ for $1 \leqslant i \leqslant|\mathfrak{C}|$ and $i \in \mathfrak{c}$ for $|\mathfrak{C}|+1 \leqslant i \leqslant|\mathfrak{C}|+|\mathfrak{c}|=N$. Denote $\left|\bar{\Psi}_{\mathfrak{C}}\right\rangle=\prod_{i=1}^{|\mathfrak{C}|}\left|\psi_{(i)}\right\rangle$ and 
$\left|\bar{\Psi}_{\mathfrak{c}}\right\rangle=\prod_{i=|\mathfrak{C}|+1}^{N}\left|\psi_{(i)}\right\rangle$; hence, we have $|\bar{\Psi}\rangle=\left|\bar{\Psi}_{\mathfrak{C}}\right\rangle \otimes\left|\bar{\Psi}_{\mathfrak{c}}\right\rangle$. Given $i^{\prime} \in \mathfrak{c}$, set the suitable operator $h_{\left(i^{\prime}\right)}^{\prime}=\bar{Z}_{\left(i^{\prime}\right)}=$ $\prod_{j^{\prime}=1}^{n_{i^{\prime}}}{\widehat{t^{\prime}}}_{\left(i^{\prime}, j^{\prime}\right)}$ that fulfills the following conditions: (i) if $\left(i^{\prime}, j^{\prime}\right) \rightarrow \mathcal{S}^{(k)}$, then ${\widehat{t^{\prime}}}_{\left(i^{\prime}, j^{\prime}\right)}=I$; and (ii) if $\left(i^{\prime}, j^{\prime}\right) \rightarrow \mathcal{R}^{(k)}$, then either qubit $\left(i^{\prime}, j^{\prime}\right)$ is idle, or $\widehat{t}_{\left(i^{\prime}, j^{\prime}\right)}=\widehat{s}_{\left(i^{\prime}, j^{\prime}\right)}$ if qubit $\left(i^{\prime}, j^{\prime}\right)$ is not idle. The logical phase-flip operator can be revised as

$$
\bar{Z}_{\left(i^{\prime}\right)}=\prod_{j^{\prime},\left(i^{\prime}, j^{\prime}\right): \text { :on the source side }} \widehat{s}_{\left(i^{\prime}, j^{\prime}\right)} \prod_{j^{\prime \prime},\left(i^{\prime}, j^{\prime \prime}\right): \text { idle }}{\widehat{o^{\prime}}}_{\left(i^{\prime}, j^{\prime \prime}\right)},
$$

where $\widehat{o}_{\left(i^{\prime}, j^{\prime \prime}\right)} \in\left\{\widehat{o}_{\left(i^{\prime}, j^{\prime \prime}\right)}, I\right\}$. Instead of measuring $B_{0}^{(k)}$ directly, the agent $\mathcal{R}^{(k)}$ measures

$$
\bar{B}_{0}^{(k)}=B_{0}^{(k)} \prod_{\substack{\left.\left(i^{\prime}, j^{\prime}\right) \rightarrow \mathcal{R}^{(k)} \\ \widehat{s}_{\left(i^{\prime}, j^{\prime}\right)}\right)=I}}{\widehat{t^{\prime}}}_{\left(i^{\prime}, j^{\prime}\right)} .
$$

The outcome of $B_{0}^{(k)}$ can be obtained from that of $\bar{B}_{0}^{(k)}$ by dropping any outcome of the local observables ${\widehat{t^{\prime}}}_{\left(i^{\prime}, j^{\prime}\right)}$ in Eq. (41); the outcome of $\prod_{i^{\prime}, i^{\prime} \in \mathfrak{c}} \bar{Z}_{\left(i^{\prime}\right)}$ can be obtained from that of $\prod_{k=1}^{K} \bar{B}_{0}^{(k)}$ by dropping any outcome of the qubit $\left(i^{\prime}, j^{\prime \prime}\right)$ if (i) $i^{\prime} \in \mathfrak{C}$ or (ii) $i^{\prime} \in \mathfrak{c}$ and $\widehat{o}_{\left(i^{\prime}, j^{\prime \prime}\right)}$ in Eq. (40) is the unit matrix. In this case, we propose the tilted Bell inequalities tailored for $|\bar{\Psi}\rangle$

$$
G_{N, K}^{\beta}=\beta\left|P_{N, K}\right|^{\frac{1}{K}}+\left|I_{N, K}\right|^{\frac{1}{K}}+\left|J_{N, K}\right|^{\frac{1}{K}} \stackrel{c}{\leqslant} \beta+1,
$$

where $P_{N, K}=\prod_{i=1}^{|\mathfrak{C}|} I^{\otimes n_{i}} \prod_{i^{\prime}=|\mathfrak{C}|+1}^{N} \bar{Z}_{\left(i^{\prime}\right)}$. However, it is very difficult to exploit sum-of-squares decomposition to find the tailored Bell operators in quantum networks with an extremely large Hilbert space [16,34]. Our strategy is to simplify $G_{N, K}^{\beta}$ as tilted CHSH inequalities. In detail, according to Lemma 2, we have

$$
\begin{aligned}
& G_{N, K}^{\beta} \leqslant \bar{G}_{N, K}(\beta, \bar{\phi}, \bar{\theta})=\beta(\cos 2 \bar{\phi})^{\frac{|c|}{K}} \\
& \quad+\cos \bar{\theta}+\sin \bar{\theta}(\sin 2 \bar{\phi})^{\frac{|c|}{K}},
\end{aligned}
$$

where equality holds when $\theta_{1}=\ldots=\theta_{N}=\bar{\theta}$. Let the parameter $\beta$ satisfying $\left.\frac{\partial}{\partial \bar{\theta}} \bar{G}_{N, K}\right|_{\left(\bar{\theta}_{\text {max }}, \bar{\phi}\right)}=\left.\frac{\partial}{\partial \bar{\phi}} \bar{G}_{N, K}\right|_{\left(\bar{\theta}_{\text {max }}, \bar{\phi}\right)}=0$ be $\beta_{\max }$. We have

$$
\tan \bar{\theta}_{\max }=(\sin 2 \bar{\phi})^{\frac{|c| c \mid}{K}}
$$

and

$$
\beta_{\max }=\frac{(\tan 2 \bar{\phi})^{\frac{2|c|}{K}-2}}{\sqrt{\left(1+\tan ^{2} 2 \bar{\phi}\right)^{\frac{|c|}{K}}+(\tan 2 \bar{\phi})^{\frac{2|c| c \mid}{K}}} .}
$$

As a result, the state $\left|\bar{\Psi}_{\mathfrak{C}}\right\rangle \otimes\left|\bar{\Psi}_{\mathfrak{c}}\right\rangle$ can maximally violate the tailored Bell inequality $G_{N, K}^{\beta_{\max }}=\bar{G}_{N, K}\left(\beta_{\max }{ }^{c} \bar{\phi}, \bar{\theta}_{\max }\right) \leqslant$ $\beta_{\max }+1$ by setting $\theta_{1}=\ldots=\theta_{N}=\bar{\theta}_{\max }$. Note that $G_{N, K}^{\beta_{\max }}$ coincides with the tilted $\mathrm{CHSH}$ inequality in the case that $\frac{|\mathfrak{c}|}{K}=1[16]$.

As an example, we consider the following "starlike" quantum network, as shown in Fig. 2. Here, set $K=N$ as an odd integer and $M=1$. For any $i$, let $\left|\psi_{(i)}\right\rangle=\cos \phi_{i}\left|\overline{0}_{i}\right\rangle+$ $\sin \phi_{i}\left|\overline{1}_{i}\right\rangle$ be the codeword of the $[5,1,3]$ QECC, where $i \in \mathfrak{C}$ if $\phi_{i}=\frac{\pi}{4}$ and $i \in \mathfrak{c}$ if $\phi_{i}=\bar{\phi} \neq \frac{\pi}{4}$. The useful operators are $g_{(i)}=\mathfrak{g}_{4}, h_{(i)}=\bar{X}_{(i)}^{\prime}$, and $h_{(i)}^{\prime}=\bar{Z}_{(i)}=\mathfrak{g}_{1} \mathfrak{g}_{3} \bar{Z}_{(i)}^{\prime}=$ $-I_{(i, 2)} X_{(i, 3)} X_{(i, 4)} I_{(i, 5)} Z_{(i, 1)}$. On the source side, agent $\mathcal{S}^{(i)}$ possesses qubit $(i, 2)$, and using the cut-and-mix method, the local observables are set as $A_{x_{i}}^{(i)}=\cos \theta_{i} Z_{(i, 2)}+(-1)^{x_{i}} \sin \theta_{i} X_{(i, 2)}$ for any $i=1, \ldots, N$. On the receiving side, the only agent $\mathcal{R}^{(1)}$ possesses qubits $(j, 1),(j, 3),(j, 4)$, and $(j, 5), 1 \leqslant$ $j \leqslant N$. Notably, ${\widehat{t^{\prime}}}_{(j, 2)}=I,{\widehat{t^{\prime}}}_{(j, 1)}=\widehat{s}_{(j, 1)}=\sigma_{z}$, and qubits $(j, 3),(j, 4)$, and $(j, 5)$ are idle. Here, two local observables for $\mathcal{R}^{(1)}$ are $B_{0}^{(1)}=\prod_{j=1}^{N} X_{(j, 3)} I_{(j, 4)} X_{(j, 5)} Z_{(j, 1)}$ and $B_{1}^{(1)}=$ $\prod_{j=1}^{N} X_{(j, 3)} X_{(j, 4)} X_{(j, 5)} X_{(j, 1)}$. In this case, one can set $\bar{B}_{0}^{(1)}=$ $-B_{0}^{(1)} \prod_{j=1}^{N} X_{(j, 4)}$. In practice, $\mathcal{R}^{(1)}$ randomly measures $\sigma_{x}$ or $\sigma_{z}$ on the qubits $(j, 1), \ldots,(N, 1)$ and always measures $\sigma_{x}$ on qubits $(j, 3),(j, 4)$, and $(j, 5), 1 \leqslant j \leqslant N$. In this scenario, the numerical simulation shows that $\max G_{N, K}^{\beta}=\beta_{\max }$.

\section{CONCLUSIONS}

In conclusion, we study the quantum networks with sources emitting different stabilizer states. To characterize Bell nonlocality, knowledge of the emitted entangled states is demonstrated to be quite useful. Regarding qubit distributions in quantum networks, nonlinear Bell inequalities are proposed which can reveal variant facets of Bell nonlocality. On the other hand, by fully exploiting the logical bit-flip and phaseflip operators, we derive tilted nonlinear Bell inequalities tailored for the codewords of $[5,1,3]$ QECC with a specific qubit distribution. It is interesting to construct the tilted nonlinear Bell inequalities maximally violated by $|\bar{\Psi}\rangle$ comprising the generic codewords of QECCs in quantum networks.
[1] J. S. Bell, On the Einstein-Podolsky-Rosen paradox, Physics 1, 195 (1964).

[2] A. Einstein, B. Podolsky, and N. Rosen, Can quantummechanical description of physical reality be considered complete? Phys. Rev. 47, 777 (1935).

[3] S. Pironio, A. Acín, S. Massar, A. Boyer de la Giroday, D. N. Matsukevich, P. Maunz, S. Olmschenk, D. Hayes, L. Luo, T. A. Manning, and C. Monroe, Random numbers certified by Bell's theorem, Nature 464, 1021 (2010).

[4] A. Acín, N. Brunner, N. Gisin, S. Massar, S. Pironio, and V. Scarani, Device-Independent Security of Quantum Cryptogra- phy Against Collective Attacks, Phys. Rev. Lett. 98, 230501 (2007).

[5] H. Buhrman, R. Cleve, S. Massar, and R. de Wolf, Nonlocality and communication complexity, Rev. Mod. Phys. 82, 665 (2010).

[6] M. Hein, J. Eisert, and H. J. Briegel, Multiparty entanglement in graph states, Phys. Rev. A 69, 062311 (2004).

[7] L.-Y. Hsu, Bell-type inequalities embedded in the subgraph of graph states, Phys. Rev. A 73, 042308 (2006).

[8] A. Cabello, O. Gühne, and D. Rodríguez, Mermin inequalities for perfect correlations, Phys. Rev. A 77, 062106 (2008). 
[9] V. Scarani, A. Acín, E. Schenck, and M. Aspelmeyer, Nonlocality of cluster states of qubits, Phys. Rev. A 71, 042325 (2005).

[10] M. Plesch and V. Bužek, Entangled graphs: bipartite entanglement in multiqubit systems, Phys. Rev. A 67, 012322 (2003).

[11] O. Gühne, G. Tóth, P. Hyllus, and H. J. Briegel, Bell Inequalities for Graph States, Phys. Rev. Lett. 95, 120405 (2005).

[12] O. Gühne, A. Cabello, Generalized Ardehali-Bell inequalities for graph states, Phys. Rev. A 77, 032108 (2008).

[13] G. Wang and M. Ying, Multipartite unlockable bound entanglement in the stabilizer formalism, Phys. Rev. A 75, 052332 (2007).

[14] L.-Y. Hsu, Mixed entangled states with two or more common stabilizers, Phys. Rev. A 76, 022322 (2007).

[15] A. Acín, S. Massar, and S. Pironio, Randomness Versus Nonlocality and Entanglement, Phys. Rev. Lett. 108, 100402 (2012).

[16] C. Bamps and S. Pironio, Sum-of-squares decompositions for a family of Clauser-Horne-Shimony-Holt-like inequalities and their application to self-testing, Phys. Rev. A 91, 052111 (2015).

[17] A. Tavakoli, P. Skrzypczyk, D. Cavalcanti, and A. Acín, Nonlocal correlations in the star-network configuration, Phys. Rev. A 90, 062109 (2014).

[18] A. Tavakoli, Bell-type inequalities for arbitrary noncyclic networks, Phys. Rev. A 93, 030101(R) (2016).

[19] R. Chaves, Polynomial Bell Inequalities, Phys. Rev. Lett. 116, 010402 (2016).

[20] M.-X. Luo, Computationally Efficient Nonlinear Bell Inequalities for Quantum Networks, Phys. Rev. Lett. 120, 140402 (2018).

[21] C. Branciard, D. Rosset, N. Gisin, and S. Pironio, Bilocal versus nonbilocal correlations in entanglement-swapping experiments, Phys. Rev. A 85, 032119 (2012).

[22] C. Branciard, N. Brunner, H. Buhrman, R. Cleve, N. Gisin, S. Portmann, D. Rosset, and M. Szegedy, Classical Simulation of
Entanglement Swapping with Bounded Communication, Phys. Rev. Lett. 109, 100401 (2012).

[23] M.-X. Luo, Nonlocality of all quantum networks, Phys. Rev. A 98, 042317 (2018).

[24] F. Andreoli1, G. Carvacho, L. Santodonato, R. Chaves, and F. Sciarrino, Maximal qubit violation of $n$-locality inequalities in a star-shaped quantum network, New J. Phys. 19, 113020 (2017).

[25] R. Cleve, D. Gottesman, and H.-K. Lo, How to Share a Quantum Secret, Phys. Rev. Lett. 83, 648 (1999).

[26] P. W. Shor and J. Preskill, Simple Proof of Security of the BB84 Quantum Key Distribution Protocol, Phys. Rev. Lett. 85, 441 (2000).

[27] A. Acín, I. Cirac, and M. Lewenstein, Entanglement percolation in quantum networks, Nat. Phys. 3, 256 (2007).

[28] K. Hammerer, A. S. Sørensen, and E. S. Polzik, Quantum interface between light and atomic ensembles, Rev. Mod. Phys. 82, 1041 (2010).

[29] N. Sangouard, C. Simon, H. de Riedmatten, and N. Gisin, Quantum repeaters based on atomic ensembles and linear optics, Rev. Mod. Phys. 83, 33 (2011).

[30] A. Mouzali, F. Merazka, and D. Markham, Quantum secret sharing with error correction, Commun. Theor. Phys. 58, 661 (2012).

[31] Z.-R. Zhang, W.-T. Liu, and Cheng-Zu Li, Quantum secret sharing based on quantum error-correcting codes, Chin. Phys. B 20, 050309 (2011).

[32] F. Baccari, R. Augusiak, I. Šupić, and A. Acín, DeviceIndependent Certification of Genuinely Entangled Subspaces, Phys. Rev. Lett. 125, 260507 (2020).

[33] F. Baccari, R. Augusiak, I. Šupić, J. Tura, and A. Acín, Scalable Bell Inequalities for Qubit Graph States and Robust SelfTesting, Phys. Rev. Lett. 124, 020402 (2020).

[34] D. Saha, R. Santos, and R. Augusiak, Sum-of-squares decompositions for a family of noncontextuality inequalities and self-testing of quantum devices, Quantum 4, 302 (2020). 